\title{
Distribución de temperatura y salinidad en campañas oceanográficas recientes en el Pacífico Tropical Oriental de Costa Rica
}

Rodney E. Mora-Escalante ${ }^{1,2,3^{*}}$, Omar G. Lizano ${ }^{1,3}$, Eric J. Alfaro ${ }^{1,2,4}$ \& Alejandro Rodríguez ${ }^{1,3}$

1. Centro de Investigación en Ciencias del Mar y Limnología (CIMAR), Ciudad de la Investigación, Universidad de Costa Rica, San José, Costa Rica; rodney.moraescalante@ucr.ac.cr, omarglizano@gmail.com, alejandro.rodriguez_b@ucr.ac.cr

2. Escuela de Física, Sede Rodrigo Facio, Universidad de Costa Rica, San José, Costa Rica

3. Módulo de Información Oceanográfica (MIO), Centro de Investigación en Ciencias del Mar y Limnología, Ciudad de la Investigación, Universidad de Costa Rica, San José, Costa Rica.

4. Centro de Investigaciones Geofísicas (CIGEFI), Ciudad de la Investigación, Universidad de Costa Rica, San José, Costa Rica; erick.alfaro@ucr.ac.cr

* Correspondence

Recibido 24-I-2019. Corregido 26-VI-2019. Aceptado 27-VII-2019.

\begin{abstract}
Distribution of temperature and salinity during recent oceanographic campaigns in the Eastern Tropical Pacific of Costa Rica. Introduction: In Costa Rica, studies done in the Eastern Tropical Pacific or ETP are important because of their interaction/relationship with the Eastern Tropical Pacific Seascape, a known multinational conservation initiative. The present study describes the physical parameters of sea temperature and salinity around Cocos Island and its environment, the ETP, using CTD profiles. Methods: Data were obtained in April 2008, March 2009, April 2010, July 2011 and March 2012 near this island. Latitudinal and longitudinal transects were also done during October 2010 and March 2011 in a broader Costa Rican ETP region. In July 2012, three latitudinal transects were done around Cocos Island. Records were obtained using a CTD, measuring conductivity, temperature and pressure, among other variables. Results: Tropical Surface Water was observed in the upper $50 \mathrm{~m}$, showing temperatures above $25^{\circ} \mathrm{C}$ and salinities below 33psu. Below the surface waters, Subsurface Subtropical Water was detected below a $60 \mathrm{~m}$ depth, showing temperatures colder than $25^{\circ} \mathrm{C}$ and salinities around $35 \mathrm{psu}$. The transition between these waters was around $50-60 \mathrm{~m}$ in depth. Temperature and salinity results were related with the atmosphere conditions observed, in which ETP variability was strongly influenced by the Inter Tropical Convergence Zone migration. Conclusions: This study also showed that reanalysis data from the HYCOM-NCODA general circulation model are comparable and in a good agreement with the CTD profiles. Temperature and salinity HYCOM data also reproduced, in general terms, the main characteristics of CTD data, having some small differences in the deeper levels. This reanalysis data set could also be used to study the tropical ocean dynamics, in wider ETP regions and for different seasons.
\end{abstract}

Key words: Sea temperature; salinity; vertical and horizontal distributions; CTD profiles; Cocos Island; Eastern Tropical Pacific.

Mora-Escalante, R. E., Lizano, O. G., Alfaro, E. J., \& Rodríguez, A. (2020).Distribución de temperatura y salinidad en campañas oceanográficas recientes en el Pacífico Tropical Oriental de Costa Rica. Revista de Biología Tropical, 68(Supl. 1), S177-S197.

Para Costa Rica, este estudio es importante ya que aporta información oceanográfica básica en una región en donde dichos estudios son escasos, ya que el mismo se realizó dentro del área que comprende el Corredor Marino del Pacífico Tropical Este (Peñaherrera et al., 2018), un esfuerzo de conservación multinacional apoyado originalmente por áreas 
marinas protegidas en Costa Rica (Isla del Coco), Panamá (Coiba), Ecuador (Galápagos) y Colombia (Mal Pelo y Gorgona), que se pretende extender a otras áreas en México (Revillagigedo) y territorios ultramarinos de Francia (Clipperton). Investigaciones de la dinámica del Pacífico Tropical del Este (PTE) comenzaron con los trabajos de Wyrtki (1964; 1966; 1967) y ampliados luego por Brenes (1984); Kessler (2006); Fiedler \& Talley (2006); Amador, Alfaro, Lizano, \& Magaña (2006); Willett, Leben, \& Lavín (2006); Lizano (2008; 2016); Amador, Rivera, Durán-Quesada, Mora, Sáenz, Calderón \& Mora (2016a) y Amador, DuránQuesada, Rivera, Mora, Sáenz, Calderón \& Mora (2016b). De estos trabajos se resume que la dinámica de la región está ligada a los fuertes vientos que atraviesan los pasos topográficos de Tehuantepec al sur de México, el Golfo de Papagayo en el Pacífico norte de Costa Rica y el Golfo de Panamá. Además una componente de la dinámica de esta región se ve influenciada por los remolinos de mesoescala que pasan, acarreando propiedades costeras (Trasviña, Barton, Brown, Velez, Kosro \& Smith, 1995; Palacios \& Bograd, 2005; Ballestero \& Coen, 2004; Trasviña \& Barton, 2008; Barton, Lavín \& Trasviña, 2009; Flores-Vidal, Durazo, Chavanne, \& Flament, 2011; Reyes-Hernández, Ahumada-Sempoal \& Durazo, 2016). La variación estacional del viento y la oscilación meridional de la Zona de Convergencia Intertropical (ZCIT) (e.g. Quirós, 2015; Hidalgo, Durán-Quesada, Amador \& Alfaro, 2015; Quirós \& Hidalgo, 2016), modifican las corrientes superficiales, la temperatura superficial del mar, la salinidad, el oleaje, entre otras variables asociadas a la dinámica en el PTE (Kessler, 2006). También se ha encontrado influencia de corrientes superficiales y sub-superficiales del sur y del oeste en esta zona (Wyrtki, 1967; Brenes, 1984; Brenes \& Coen, 1985; Lizano, 2008; 2016), siendo El Niño-Oscilación del Sur o ENOS la principal fuente de variabilidad interanual que modula el clima en el PTE (Amador et al., 2016a).

Los perfiles en la columna de agua de temperatura, salinidad y oxígeno disuelto que se hicieron alrededor del Domo Térmico de Costa Rica o DTCR (Lizano, 2016; Bronnkow, 1965), muestran las condiciones típicas que deberían encontrarse en las aguas tropicales: poca variación estacional y gradientes fuertes en la capa superficial. Las mediciones realizadas por Brenes, Ballestero, Benavides, Salazar \& Murillo (2016) a lo largo de un transecto norte-sur $\left(10^{\circ}\right.$ a $\left.3.5^{\circ} \mathrm{N}\right)$ realizado en octubre del 2010 y marzo del 2011, muestran que hay una termoclina menos profunda al norte que al sur. En salinidad, valores menores se encontraron al norte cuando los mismos son comparados con la parte sur, en ese transecto de octubre de 2010. En marzo 2011 el transecto es menos homogéneo (Brenes et al., 2016).

En el PTE los perfiles en la columna de agua de la temperatura y la salinidad, están relacionados con procesos, tanto oceánicos, como atmosféricos (Brenes et al., 2016; Lizano, 2016; Amador et al., 2016a;b). Así como el viento local genera corrientes marinas importantes en esta zona, también genera enfriamientos superficiales, los cuales modifican la estructura vertical de la temperatura (Brenes, 1984; Fiedler, 2002; Kessler, 2006; Alfaro \& Cortés, 2012; Alfaro, Cortés, Alvarado, Jiménez, León, Sánchez-Noguera, Nivia-Ruiz, \& Ruiz, 2012). Por otro lado, los procesos atmosféricos, como la alta radiación solar y precipitación que se dan en estas latitudes (Amador et al., 2006; 2016a;b), también generan gradientes fuertes en la distribución espacial de estos parámetros y en la columna de agua (Lizano, 2013; 2016). Durante el invierno boreal los vientos intensos sobre el Golfo de Papagayo y el Golfo de Panamá, mantienen enfriamientos superficiales en estas regiones (Fielder, 2002; Alfaro \& Cortés 2012; Alfaro et al., 2012). El enfriamiento superficial de Papagayo es aún observable hacia los $6^{\circ} \mathrm{N}$ y $95^{\circ} \mathrm{W}$ y condicionan la distribución superficial de la temperatura y la salinidad (Lizano, 2016). La surgencia de aguas desde capas sub-superficiales, implica aguas más frías, más salinas y con menor concentración de oxígeno disuelto (Lizano, 2016; Fiedler, 2006; Kessler, 2006; Brenes, 1985), las cuales como lo menciona Wyrtki (1964), 
vienen desde capas debajo de la termoclina $(75-200 \mathrm{~m})$, donde las temperaturas rondan los $12-14^{\circ} \mathrm{C}$.

El análisis hecho por Lizano (2016), muestra que la variación climática mensual de los perfiles de temperatura y salinidad en la columna de agua, se da sobre los $200 \mathrm{~m}$ de profundidad y sus variaciones son, principalmente, una consecuencia de los procesos atmosféricos y oceánicos que se mencionaron al principio de esta sección. La marcha mensual de los gradientes con profundidad de estos perfiles depende de la variación espacial e intensidad de los enfriamientos superficiales generados en esta zona.

Caracterizaciones de las masas de agua en el PTE fueron realizadas por Wyrtki (1967), a través de correlaciones de Temperatura y Salinidad (diagramas T-S). Brenes (1985) basado en los diagramas T-S del trabajo de Wyrtki (1967), encuentra que para esta región están presentes el Agua Superficial Tropical (AST), Agua Sub-superficial Sub-tropical (ASS) y el Agua Intermedia Antártica (AIA). En un trabajo posterior, Brenes \& Coen (1985), comprueban que en aguas de una región del PTE, coexisten la AST, la ASS y la AIA, y que además, propiamente alrededor del DTCR, aguas de origen sub-superficial (ASS), penetran la capa superficial evidenciando un enfriamiento superficial en esta zona. El estudio más reciente de Brenes et al. (2016), de las características T-S de dos transectos del PTE a través de la Isla del Coco, vuelve a ratificar la presencia de dos masas de aguas distinguibles en esta zona: la AST y la ASS.

El AST se caracteriza en esta región del PTE por poseer salinidades menores a 34ups y temperaturas mayores a $25^{\circ} \mathrm{C}$ (Brenes, Kwiecinski, D’Croz \& Chávez, 1995), debido a que existe un exceso de precipitación sobre la evaporación en esta región y una fuerte incidencia de radiación solar a lo largo del año (Badan, 1997; Amador et al. 2016a; b). Brenes (1984) describe que el límite sur de esta agua se extiende del Ecuador en dirección de las Islas Galápagos y continua hacia el oeste hasta cerca de los $4^{\circ} \mathrm{N}$, donde coincide aproximadamente con el límite sur de la Contracorriente ecuatorial. El límite norte se puede identificar por la isoterma de $25^{\circ} \mathrm{C}$, la cual permanece cerca de $\operatorname{los} 15^{\circ} \mathrm{N}$ y oscila durante el año hasta más o menos los $5^{\circ} \mathrm{N}$, la extensión vertical de esta masa está limitada por la capa de mezcla, que alcanza usualmente los $50 \mathrm{~m}$ de profundidad. Brenes (1985), agrega que la extensión antes descrita del AST, es sensible a las variaciones estacionales de los procesos meteorológicos, principalmente; y oceanográficos. Esto fue observado por Brenes et al. (1995) en diferentes campañas realizadas en el PTE.

El ASS, posee salinidades entre 34.9 y 35 ups y una temperatura del orden de los $13^{\circ} \mathrm{C}$. Generalmente se ubica entre los 50 y $200 \mathrm{~m}$ de profundidad. Esta está separada del AST por la presencia en la región ecuatorial de una termoclina intensa (Badan 1997). Según Brenes (1984); Brenes et al. (1995) y Badan (1997), esta agua se origina en el Pacífico Sur, penetrando al PTE a través del ecuador en el hemisferio norte. Cuando alcanza el ecuador, es influenciada por la Sub-corriente Ecuatorial y pasa por procesos de mezcla con aguas cuya $\mathrm{T}$ y $\mathrm{S}$ son menores a $16^{\circ} \mathrm{C}$ y 35 ups. Brenes \& Coen (1985) agregan que esta agua puede llegar muy cerca de la superficie en regiones de enfriamiento superficial como el DTCR.

Por último, el agua intermedia antártica (AIA), posee salinidades mayores a 34.55 ups y $\mathrm{T}$ menores a $5^{\circ} \mathrm{C}$ y se ubica en profundidades entre los 900 y $1000 \mathrm{~m}$. Según Brenes (1984) y Brenes et al. (1995), se forma cerca del frente polar antártico y se extiende en dirección norte, razón por la cual su salinidad y $\mathrm{T}$ van aumentando con la latitud.

El presente estudio pretende la descripción de los parámetros físicos de temperatura y salinidad del océano para las masas de agua que rodean a la Isla del Coco y su entorno, el PTE, a través del análisis de perfiles de CTD obtenidos durante campañas hidrográficas realizadas entre el año 2008 y el 2012. Adicionalmente se pretende enmarcar esta descripción, dentro de la variabilidad climática estacional propia de la región del PTE, tal y como lo sugiere Brenes (1985). En este trabajo se amplía lo presentado 
por Brenes et al. (2016) para las campañas hidrográficas de octubre de 2010 y marzo de 2011, al incluir además la realizada en julio de 2012 y comparar los resultados de los datos insitu con los del reanálisis del modelo HYCOMNCODA. También se incluye el análisis a los cruceros hechos alrededor de la Isla del Coco en abril de 2008, marzo de 2009, abril de 2010, julio de 2011 y marzo de 2012. Los detalles del área de estudio, las campañas y del reanálisis se describen en la siguiente sección.

\section{MATERIALES Y MÉTODOS}

Área de estudio: La región de estudio comprende el PTE, enfocado en las aguas que rodean a la Isla del Coco, Costa Rica. La Isla del Coco está ubicada aproximadamente a $500 \mathrm{~km}$ al SSW de Cabo Blanco, Puntarenas, el punto más cercano a Costa Rica y alrededor de $\operatorname{los} 87^{\circ} \mathrm{W}$ y $5.5^{\circ} \mathrm{N}$ (Wyrtki 1967; Lizano 2001; Kessler 2006). En la Fig. 1 se muestra el área de interés para el presente estudio y se puede observar la localización de las diferentes estaciones hidrográficas que se realizaron para obtener un perfil de la columna de agua, en aguas profundas o lejos de la costa (Fig. 1A) y en aguas someras alrededor de la isla (Fig. 1B). Los detalles batimétricos de la región pueden ser encontrados en Lizano (2001; 2008).

Campañas de muestreo: Se compiló una base de datos oceanográficos conformada por los resultados de 8 diferentes campañas científicas a la Isla del Coco (Cortés, Morales, Alfaro, Lizano, \& Acuña, 2009; Cortés, Brenes, Morales, Lizano, Alfaro, Acuña, Ballestero, \& Moreno, 2012). Durante las campañas científicas de abril de 2008, abril de 2010, julio de 2011 y marzo de 2012 se tomaron los datos correspondientes a los transectos cerca de Isla Dos Amigos, Bahía Chatham, Isla Montagne y Bahía Wafer. En marzo de 2009 se realizaron los transectos anteriormente mencionados (excepto el de Bahía Wafer), más 3 transectos adicionales en los alrededores de Cabo Atrevida, Isla Pájara y Punta María (Fig. 1A). En las campañas de octubre de 2010 y marzo de
2011 se realizó un transecto latitudinal y un transecto longitudinal en las aguas del Pacífico Tropical Este de Costa Rica. En julio de 2012 se realizaron 3 transectos latitudinales en las aguas en torno a la Isla del Coco (Fig. 1B). Los detalles y localización de los accidentes geográficos mencionados anteriormente pueden ser consultados en Díaz-Bolaños, Alfaro \& Ugalde-Quesada (2012).

Las mediciones se realizaron por medio de un CTD modelo SBE 19plus V2, instrumento utilizado para determinar la conductividad, temperatura y presión de la columna de agua, entre otras variables. Dicho equipo fue calibrado bajo los estándares del fabricante. Los datos crudos fueron descargados del CTD y procesados mediante el software SBE Data Processing de Sea-Bird Electronics. Durante las campañas se tomaron muestras de agua por medio de botellas Nisken para la comparación con los datos de temperatura y salinidad, con resultados coherentes al compararlos con los obtenidos con el CTD. Se obtuvo un mapa para cada uno de los transectos trazados durante las distintas campañas, por lo cual se cuenta con 22 mapas de distribución vertical de transectos costeros y 7 mapas de transectos oceánicos. Los transectos costeros corresponden a estaciones de muestreos utilizando una polea manual, ubicadas dentro de trayectorias rectas en dirección a la costa de la Isla del Coco. Los transectos oceánicos están conformados por estaciones de muestreos con una polea mecánica, ubicadas dentro de trayectorias rectas en las aguas del PTE de Costa Rica. Además, las estaciones de transectos oceánicos están separadas por una mayor distancia y alcanzan una mayor profundidad en comparación a los transectos costeros, por lo que abarcan una mayor región del océano. Vale la pena aclarar que, durante la campaña oceanográfica de marzo de 2011, se utilizaron dos equipos de CTD, uno perteneciente a la Universidad de Costa Rica y otro a la Universidad Nacional de Costa Rica (Cortés et al., 2009; 2012). En el presente estudio, presentamos los resultados obtenidos con el equipo de la UCR en esa campaña. Los resultados obtenidos con el equipo de la Universidad 
A

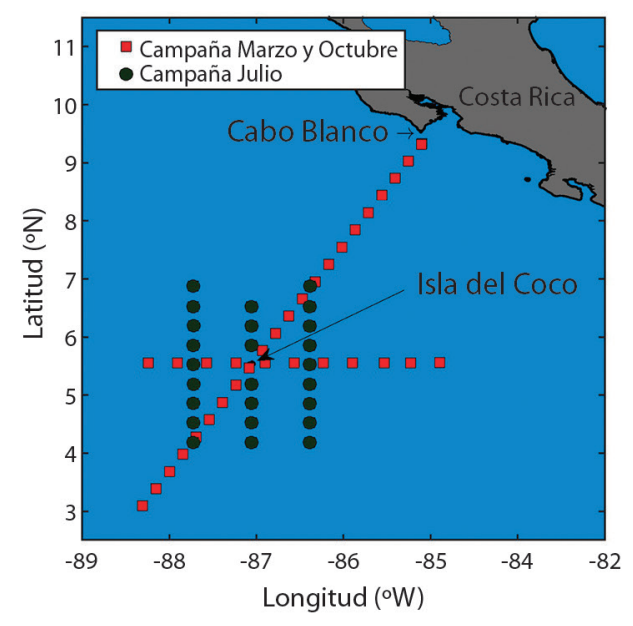

B

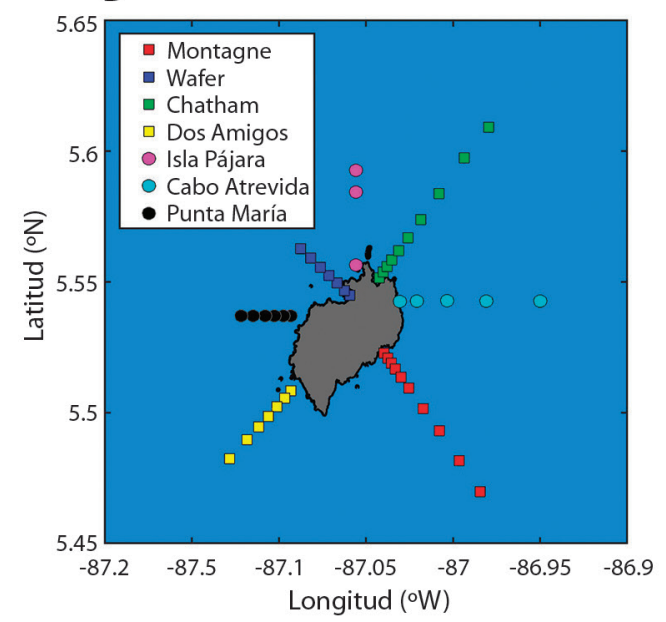

Fig. 1. Localización de las estaciones de muestreo entre los años 2008 y 2012. En A) se muestra una región sobre el Pacífico Tropical Este centrado en la Isla del Coco. Los círculos negros indican estaciones en que se registraron datos durante la campaña julio de 2012 y los círculos rojos indican estaciones con registro de datos en los meses de octubre 2010 y marzo 2011. B) Alrededores de la Isla del Coco. Los círculos representan estaciones con una sola campaña de registro de datos y los cuadros indican estaciones con al menos 4 años de registro de datos.

Fig. 1. Location of the cast stations recorded between 2008 and 2012. A) Region over the Eastern Pacific Tropical around the Cocos Island. The black circles represent cast station samplings in July 2012 and the red circles represent cast stations in the month of October 2010 and March 2011. B) Surroundings of the Cocos Island. The circles represent stations with only one cast record and the square stations with at least four years of cast records.

Nacional en marzo de 2011 se pueden consultar en Brenes et al. (2016).

Reanálisis del modelo de circulación general: Se utilizó información del reanálisis del modelo de circulación global HYCOMNCODA obtenida del sitio http://hycom.org. Este modelo emplea un sistema de asimilación de datos, NCODA (Cummings, 2005) como una componente del modelo océanico Hybrid Coordinate Ocean Model (HYCOM, Chassignet et al., 2009). El modelo tiene una resolución horizontal de $1 / 12^{\circ}$ y en la vertical tiene 42 niveles de profundidad interpolados. Cuenta con cinco variables oceanográficas globales, elevación de la superficie del mar, temperatura del agua, salinidad y el campo de velocidad de las partículas de agua. Tiene un registro de 21 años de datos con promedios diarios y cada 3 horas. Para el estudio se emplearon datos diarios de salinidad y temperatura hasta la profundidad de $400 \mathrm{~m}$ para los meses de octubre 2010, marzo 2011 y julio 2012.

\section{RESULTADOS}

Masas de agua alrededor de la Isla del Coco: Para caracterizar las masas de aguas alrededor de la Isla del Coco se presentan perfiles verticales de la distribución de temperatura y salinidad a lo largo de una trayectoria en línea recta y perpendicular a la isla. En la Fig. 2 se muestra el perfil de temperatura y salinidad del transecto para Isla Dos Amigos. Corresponde en la Fig. 1B al trayecto con cuadros de color amarillo. Las temperaturas cálidas $\left(>25^{\circ} \mathrm{C}\right)$ se mantienen alrededor de los $50 \mathrm{~m}$ de profundidad y a lo largo de los $5 \mathrm{~km}$, a excepción de la Fig. 2D, que corresponde al mes de julio, donde pareciera que hay un ascenso de aguas frías (una surgencia). Hay predominancia de una masa de agua con temperatura entre los 

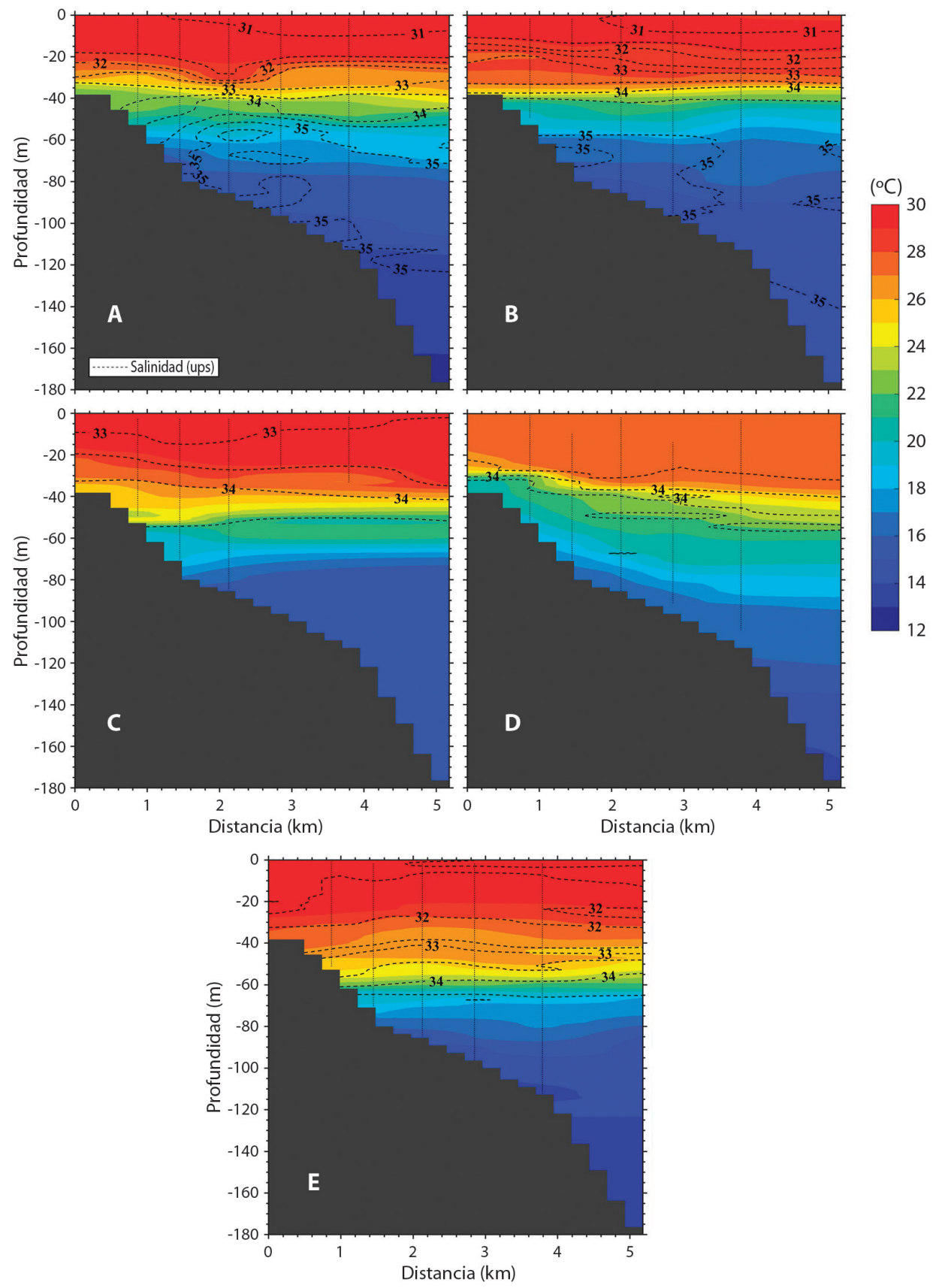

Fig. 2. Distribución vertical de temperatura y salinidad para la Isla Dos Amigos. Campañas: A) abril 2008, B) marzo 2009, C) abril 2010, D) julio 2011 y E) marzo 2012. Las mediciones corresponden al transecto con cuadros amarillos en la Fig. 1B. Los tonos de color representan la temperatura del océano. Los contornos con líneas segmentadas representan la salinidad. El intervalo de contornos es 0.5 ups y va de 30 a 38 ups. Las líneas verticales con puntos son las estaciones de muestreo.

Fig. 2. Vertical distribution of temperature and salinity for Dos Amigos Island. Campaigns: A) April 2008, B) March 2009, C) April 2010, D) July 2011 and E) March 2012. The measurements correspond to the transect with yellow squares in Fig. 1B. The colors represent the temperature of the ocean. The dashed line contours represent the salinity. The contour interval for salinity is $0.5 \mathrm{psu}$ and goes from 30 to $38 \mathrm{psu}$. The vertical lines with points are the station casts. 
$28^{\circ} \mathrm{C}$ y $30^{\circ} \mathrm{C}$ en los primeros $30 \mathrm{~m}$ (Figs. $2 \mathrm{~A}, \mathrm{~B}$, C, E), los cuales corresponden a los meses de marzo y abril. La isohalina de 35.0ups presentó poca variación en todo el periodo analizado por debajo de los 50m de profundidad. En la Fig. $2 \mathrm{~A}$ alrededor de $\operatorname{los} 2 \mathrm{~km}$ hay un incremento leve de temperatura y una disminución en la salinidad entre los 20 y $30 \mathrm{~m}$ de profundidad, lo cual podría deberse algún proceso de mezcla con agua superficial. Hay una variación en los años 2008, 2009, 2010 y 2012 de la isohalina de 33.0ups, se hunde hasta llegar aproximadamente al nivel de 50m (Fig. 2E) o se levanta hasta niveles muy cerca de la superficie (Fig. 2C).

La distribución vertical de temperatura y salinidad del transecto para Bahía Wafer se muestra en la Fig. 3 (corresponde en la Fig. $1 \mathrm{~B}$ al trayecto con cuadros de color azul). Los meses de marzo 2012 (Fig. 3D), abril 2008 (Fig. 3A) y abril 2010 (Fig. 3B), prevalece una masa de agua cálidas (una capa de mezcla), por arriba de $\operatorname{los} 28^{\circ} \mathrm{C}$, dentro de los primeros $30 \mathrm{~m}$ de profundidad. En el mes de julio 2011, hay presencia de una masa de agua con temperatura que ronda los $28^{\circ} \mathrm{C}$ en los primeros $50 \mathrm{~m}$ de profundidad (Fig. 3C) y a lo largo de los $4 \mathrm{~km}$ del transecto. También se observa que la termoclina varía entre $50 \mathrm{~m}$ a $100 \mathrm{~m}$ en profundidad. La isohalina de 34.0ups se mantiene constante sobre el nivel de 50m. En los años 2008 y 2012 de campaña hidrográfica se nota un importante gradiente de salinidad (Fig. 3A,D) en la columna de agua que se observa hasta $10 \mathrm{~s} 5 \mathrm{~m}$.

La Fig. 4 muestra el corte vertical de temperatura y salinidad del transecto para Bahía Chatham (véase Fig. 1B trayectoria de cuadros de color verde claro). Es interesante notar en la Fig. 4B (marzo 2009) como la profundidad de la termoclina se reduce conforme se aleja de la isla, con valores de $20 \mathrm{~m}$ en la costa a $10 \mathrm{~m}$ en distancias de $8 \mathrm{~km}$. Para este transecto de 2009, el gradiente de temperatura no es tan significativo como si lo es en los otros años (Figs. 4A, $\mathrm{C}, \mathrm{D}, \mathrm{E})$. Hay predominancia de aguas cálidas $\left(>27^{\circ} \mathrm{C}\right)$ en la capa superficial en los años 2008, 2009, 2010 y 2012 (Figs. 4A, B, C y E, respectivamente). Un gradiente de salinidad notorio en los años 2008, 2009 y 2012 en los primeros $60 \mathrm{~m}$ en profundidad (Figs. 4A, B, E). En la Fig. 4B es importante señalar como la isolínea de 34.0ups se levanta conforme se aleja de la línea de la costa y en la Fig. 4D como esta isohalina actúa como una división de aguas cálidas en la superficie y aguas frías en profundidad.

Para el transecto correspondiente a la Isla Montagne en la Fig. 5 se muestra el perfil de temperatura y salinidad, corresponde en la Fig. 1B a la estación con cuadros de color rojo. Resalta la alta salinidad, de 37.0ups, que se nota en la Fig. 5A para marzo de 2008 alrededor de los $100 \mathrm{~m}$ en profundidad y a una distancia de la costa de $7 \mathrm{~km}$. Esta salinidad no se presenta en los otros años de muestreo, ni para los otros transectos mostrados en la Figs. 2, 3 y 4. También es notorio el fuerte gradiente de salinidad en casi toda la columna de agua. En la Fig. 5B se presenta una región de alta salinidad (35.0ups) alrededor de los $60 \mathrm{~m}$ y localizada a $3.5 \mathrm{~km}$. Marzo 2009 y marzo 2012 presentan una haloclina que abarca hasta $10 \mathrm{~s} 60 \mathrm{~m}$. Masas de agua cálidas, mayores a $27^{\circ} \mathrm{C}$, predominan en los primeros $25 \mathrm{~m}$ de profundidad (Figs. 5A, B, C y E), excepto en la campaña del año 2011 (mes julio), ya que se observa una masa de agua superficial con un grado de temperatura menor que las mostradas para los otros años. Esta característica de su masa de agua en su distribución vertical de temperatura es consistente con lo mostrado en las Figs. 2, 3, y 4. Masas de agua más salinas se presenta en los años 2011 y 2012, en los primeros 40m en profundidad (Fig. 5D y Fig. 5E).

En la campaña del 2009 se hicieron tres transectos más, Isla Pájara, Cabo Atrevido y Punta María. En la Fig. 6 se muestran la distribución vertical de temperatura y salinidad para estos sitios (corresponden a los círculos con colores de la Fig. 1B). Las tres localizaciones presentan masas de agua cálidas en los primeros $30 \mathrm{~m}$ y que son consistentes con lo observado en las Figs. 2, 3 ,4 y 5, excepto para el mes de julio en su capa de mezcla. En Punta María (Fig. 6C) se presenta un valor máximo de salinidad alrededor de $1 \mathrm{~km}$ de la costa y cercano al fondo marino de 36.0 ups. La 


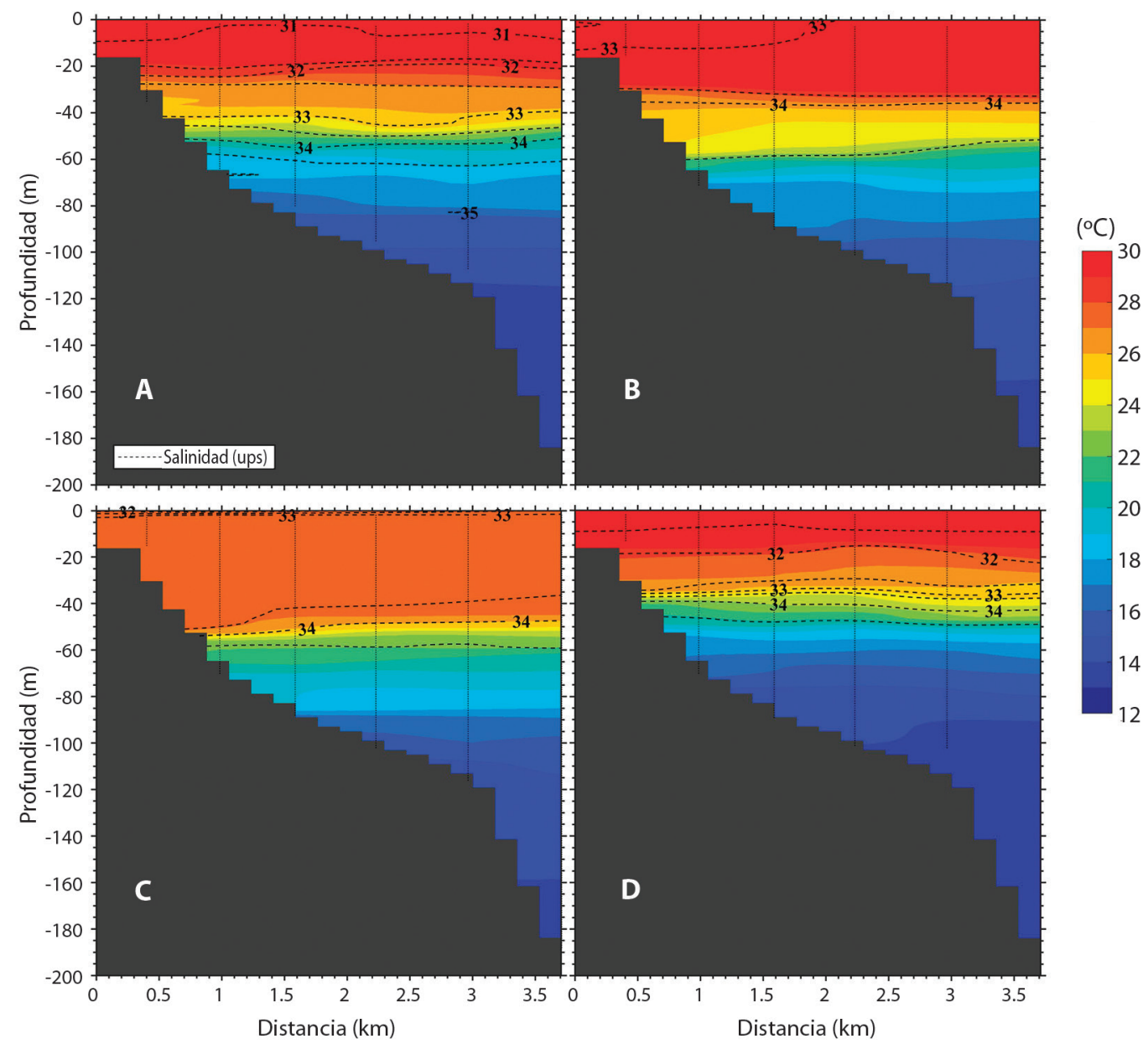

Fig. 3. Igual que en la Fig. 2 pero para Bahía Wafer. Campañas: A) abril 2008, B) abril 2010, C) julio 2011 y D) marzo 2012. Las mediciones corresponden al transecto con cuadros azules en la Fig. 1B.

Fig. 3. Same as Fig. 2 but for Wafer Bay. Campaigns: A) April 2008, B) April 2010, C) July 2011 and D) March 2012. The measurements correspond to the transect with blue squares in Fig. $1 \mathrm{~B}$.

haloclina se localiza entre los $20 \mathrm{~m}$ y $50 \mathrm{~m}$ de profundidad con las isolíneas de salinidad muy cercanas entre sí. A diferencia de la Fig. 6B (Cabo Atrevido) donde la haloclina está localizada entre los $10 \mathrm{~m}$ y $40 \mathrm{~m}$ y las isohalinas están más distanciadas. En la capa superficial de las tres figuras (Fig 6A, B y C) hay un paramétro físico en común, presentan una masa de agua con baja salinidad.

Masas de agua lejos de la Isla del Coco: Hasta ahora se ha caracterizado la distribución vertical de temperatura y salinidad cerca de la costa donde se observó la variabilidad de estos parámetros. El comportamiento lejos de la isla puede presentar un comportamiento más homógeneo en la columna de agua y diferenciando la capa superficial de la capa más profunda. Para los meses de octubre 2010, marzo 2011 y julio 2012 se hicieron una serie de transectos océanicos que se muestran en la Fig. 1A.

La Fig. 7 muestra el perfil de temperatura y salinidad del transecto realizado para el mes de octubre 2010. Consiste en dos transectos, 

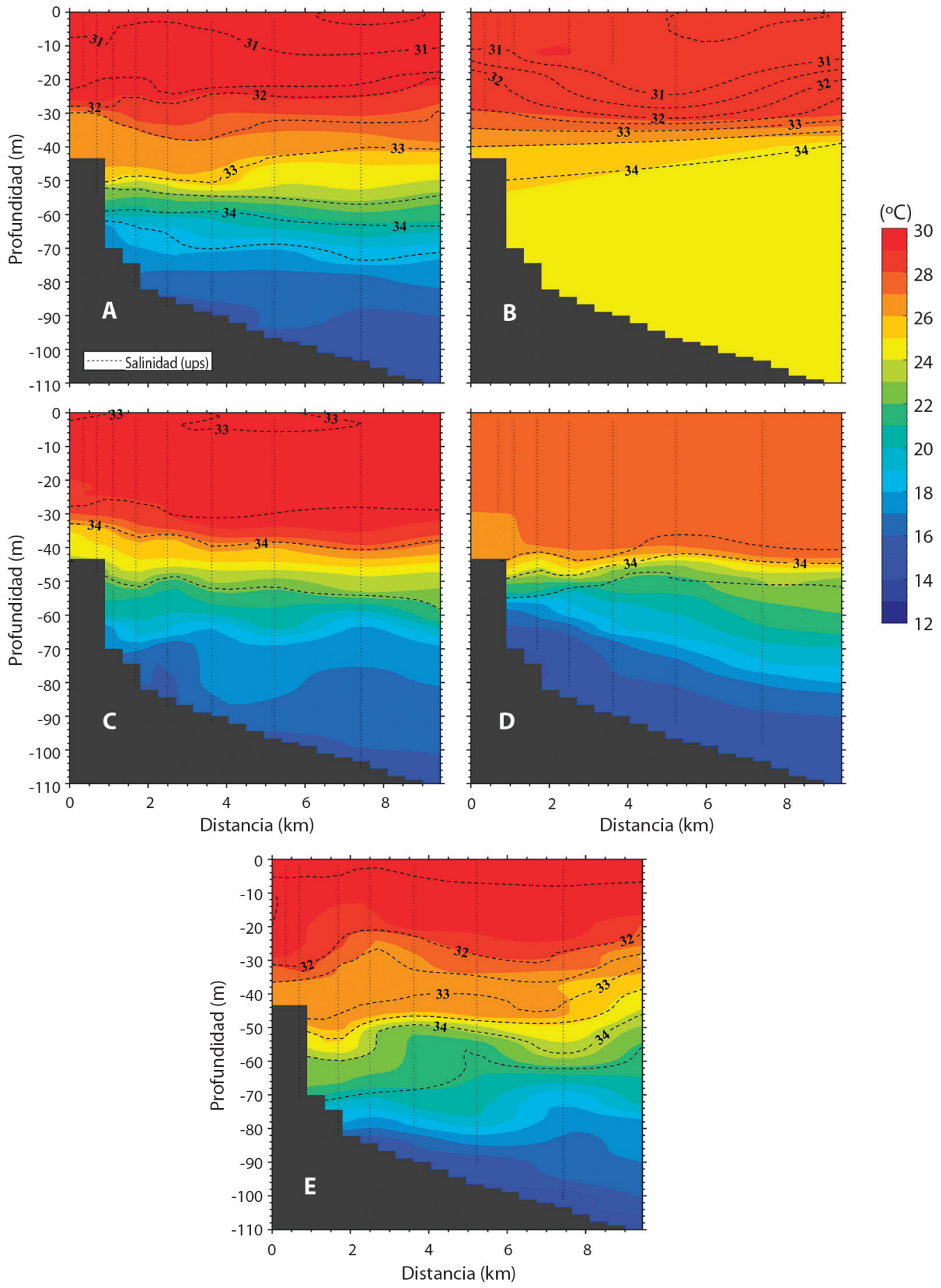

Fig. 4. Igual que en la Fig. 2, pero para Bahía Chatham. Las mediciones corresponden al transecto con cuadros verdes claros en la Fig. 1B.

Fig. 4. Same as Fig. 2, but for Chatham Bay. The measurements correspond to the transect with light green squares in Fig. 1B. 

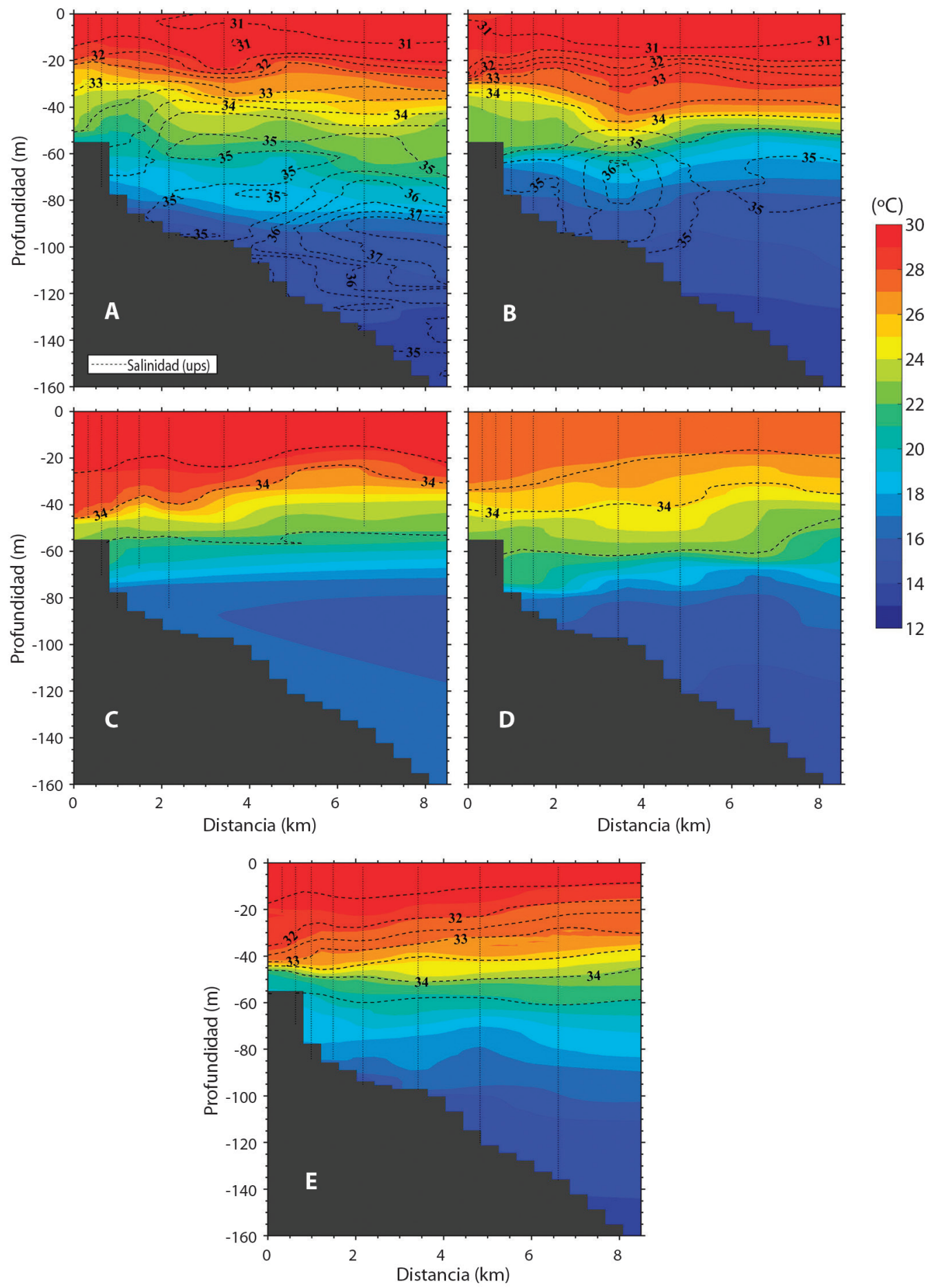

Fig. 5. Igual que en la Fig. 2, pero para la Isla Montagne. Las mediciones corresponden al transecto con cuadros rojos en la Fig. 1B.

Fig. 5. Same as Fig. 2, but for Montagne Island. The measurements correspond to the transect with red squares in Fig. 1B. 

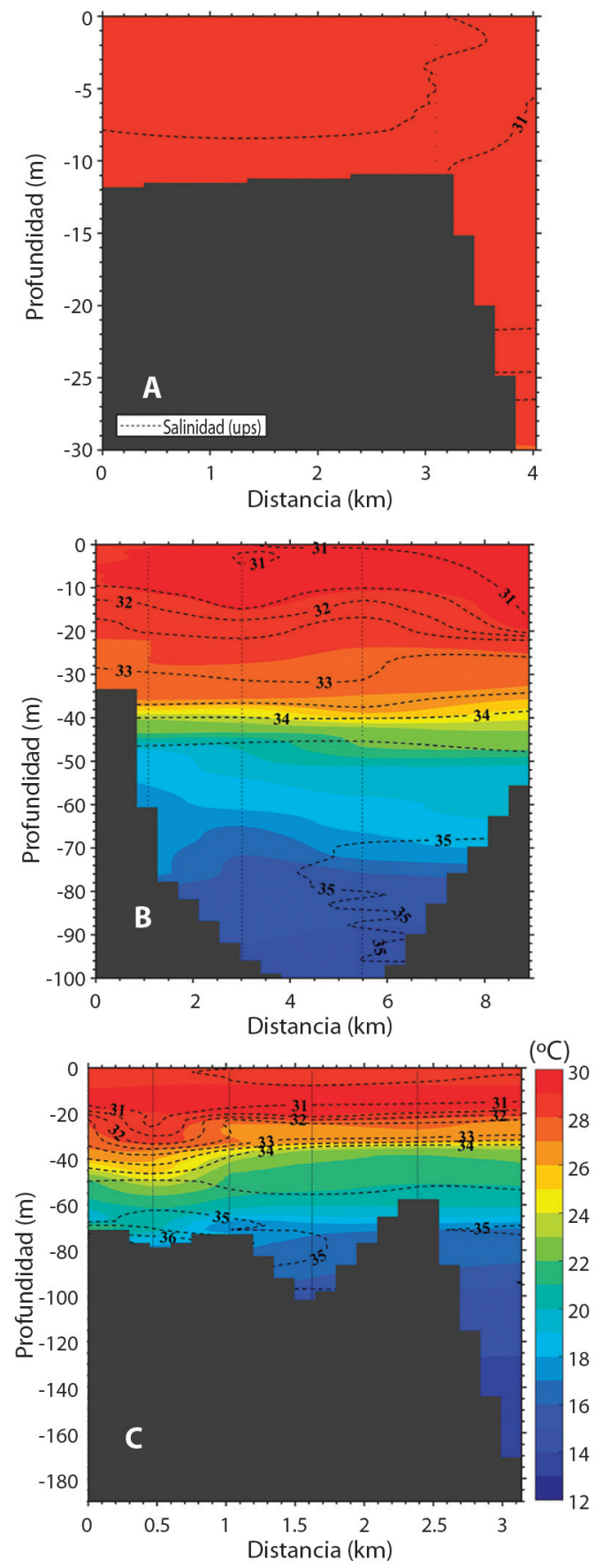

un corte vertical en latitud (Fig. 7A) y un corte vertical en longitud (Fig. 7C). Corresponden en la Fig. 1A a las estaciones con cuadros de color rojo. Para comparar con información de datos de reanálisis, se utiliza la información del modelo numérico de circulación oceánica HYCOM-NCODA. Se realiza un corte vertical en latitud (Fig. 7B) y un corte vertical en longitud (Fig. 7D) con los datos extraídos del modelo. Una capa superficial de masa de agua en el corte latitudinal (Fig. 7A) se mantiene alrededor de los $26^{\circ} \mathrm{C}$ y $27^{\circ} \mathrm{C}$ de temperatura en los primeros $40 \mathrm{~m}$, igualmente en el perfil longitudinal (Fig. 7C). La distribución de temperatura en esta capa superficial es similar a la información extraída del reanálisis (Fig. 7B y Fig. 7D). Estos resultados son consistentes con lo reportado por Brenes et al. (2016). En el corte latitudinal de las observaciones, alrededor de los $8.5^{\circ} \mathrm{N}$, se observa un núcleo de masa de agua con temperatura de $27^{\circ} \mathrm{C}$ y un levantamiento de las isohalinas hacia los $9^{\circ} \mathrm{N}$, el cual es comparable con los resultados del modelo, Fig. 7B. La termoclina se localiza entre los $40 \mathrm{~m}$ y $60 \mathrm{~m}$ en las observaciones como en los datos del modelo. Las isohalinas están más separadas en las observaciones que en el reanálisis. En el corte vertical longitudinal de los datos del modelo, específicamente en los $85.5^{\circ} \mathrm{W}$, se sitúa una masa de agua con temperatura de $27^{\circ} \mathrm{C}$ que no se presenta en las observaciones (es uniforme con una temperatura de $26^{\circ} \mathrm{C}$ ). Para el punto $87.5^{\circ} \mathrm{W}$, el valor de salinidad en las observaciones y en el modelo para la superficie, es de 33.0ups, comparable con el valor promedio de salinidad para octubre-diciembre obtenido por Lizano (2008), de 33.1ups. Para el mismo punto, en el perfil longitudinal de temperatura de las observaciones y de los datos del modelo, el valor de temperatura en la superficie, es de $26^{\circ} \mathrm{C}$, el cual es similar al valor de temperatura

Fig. 6. Igual que en la Fig. 2, pero para la campaña de marzo 2009. Transectos: A) Isla Pajara, B) Cabo Atrevida y C) Punta María. Las mediciones corresponden a los transectos con círculos fusia, turquesa y negro en la Fig. 1B, respectivamente. Fig. 6. Same as in Fig. 2 but for the campaign of March 2009. Transect: A) Pajara Island, B) Cabo Atrevida and C) Punta María. The measurements correspond to the transects with fuchsia, turquoise and black circles in Fig. 1B, respectively. 

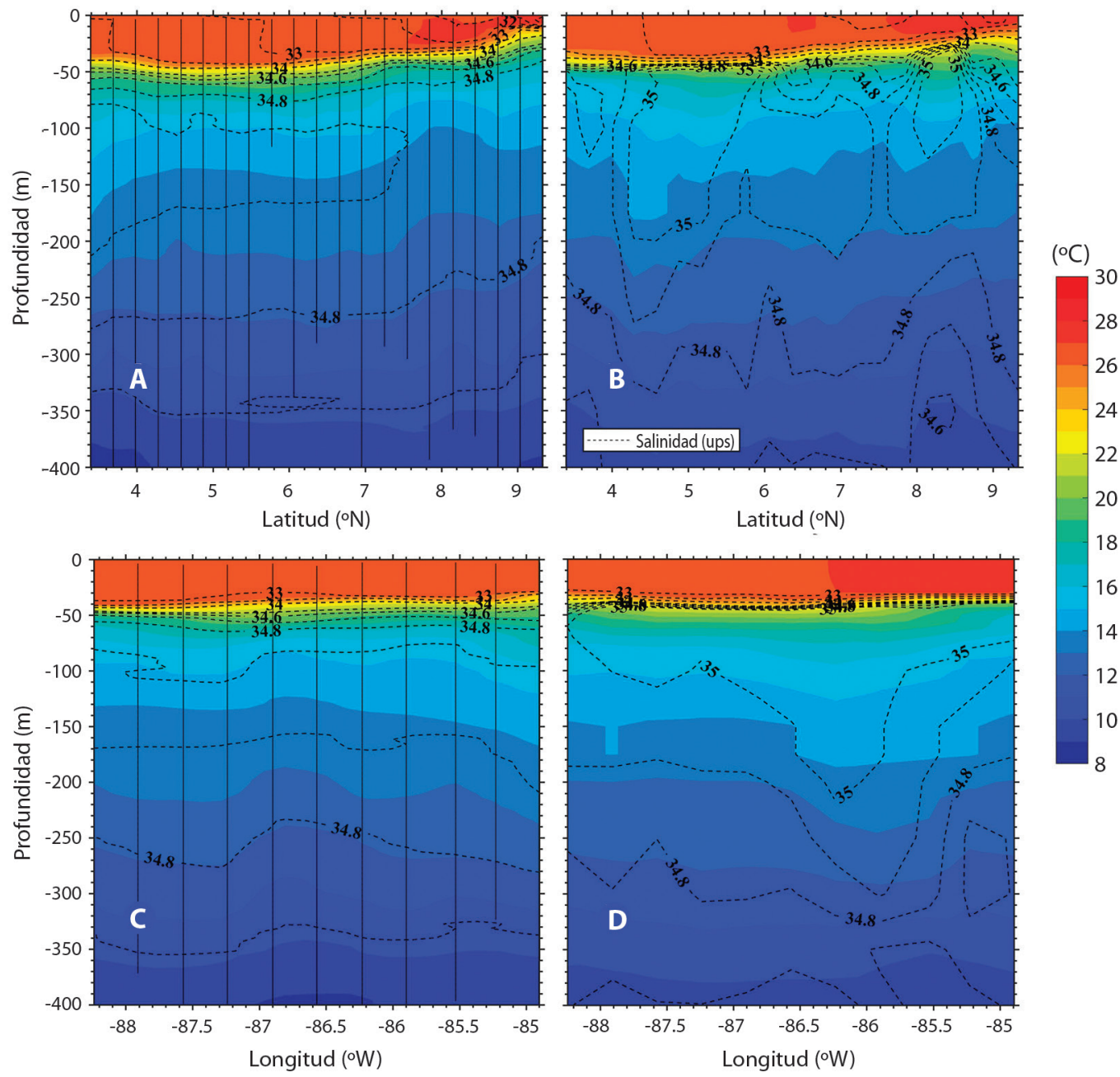

Fig. 7. Distribución vertical de temperatura y salinidad para la campaña de octubre de 2010. A) Transecto latitudinal del CTD. B) Transecto latitudinal del modelo HYCOM-NCODA reanálisis. C) Transecto longitudinal del CTD. D) Transecto longitudinal del modelo HYCOM-NCODA reanálisis. Las observaciones corresponden al transecto con cuadros rojos en la Fig. 1A. Los tonos de color representan la temperatura del océano. Los contornos con líneas segmentadas representan la salinidad. El intervalo de los contornos de salinidad es 0.5 ups en el rango de 30 a 34.5 ups y de 0.1 ups en el rango de 34.5 a 35.0ups. Las líneas verticales con puntos son las estaciones de muestreo.

Fig. 7. Vertical distribution of temperature and salinity for the campaign of October 2010. A) Latitudinal vertical section for the CTD casts. B) Latitudinal vertical section for the HYCOM-NCODA reanalysis data. C) Longitudinal vertical section for the CTD casts. D) Longitudinal vertical section for the HYCOM-NCODA reanalysis data. The observations correspond to the transect with red squares in Fig. 1A. The colors are for sea temperature. The dashed line contours are for salinity. The contour interval of salinity is $0.5 \mathrm{psu}$ from 30 to $30.4 \mathrm{psu}$ and $0.1 \mathrm{psu}$ from 34.5 to $35.0 \mathrm{psu}$. The vertical lines with points are the station casts.

promedio reportado por Lizano (2008) para el trimestre octubre-diciembre, de $26.8^{\circ} \mathrm{C}$.

Para el mes de marzo de 2011, se repitieron los mismos transectos que los de la Fig. 1A (transectos con círculo rojo). En la Fig. 8 se muestran los resultados de la distribución vertical de temperatura y salinidad para la campaña 2011. En las observaciones para el perfil latitudinal, entre los $7^{\circ} \mathrm{N}$ y $9^{\circ} \mathrm{N}$, pareciera manifestarse un evento de surgencia (Fig. 8A) 

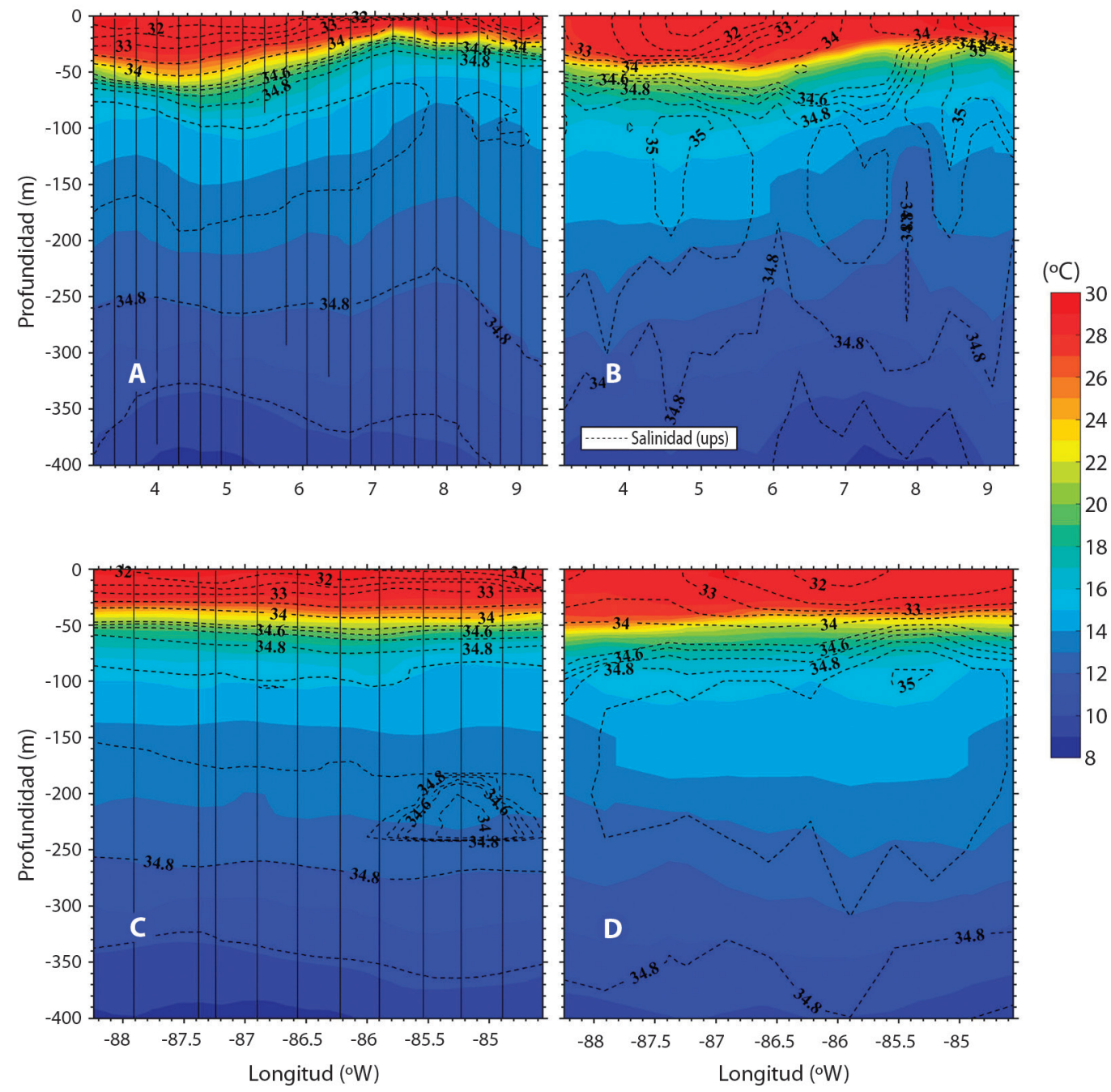

Fig. 8. Igual que en la Fig. 7, pero para la campaña de marzo de 2011.

Fig. 8. Same as Fig. 7, but for the campaign of March 2011.

comparable con lo mostrado en los datos del reanálisis (Fig. 8B). Aguas cálidas $\left(>27^{\circ} \mathrm{C}\right)$ se presentan en las observaciones como en la información del modelo. A partir de los $50 \mathrm{~m}$ empieza a descender la temperatura en las observaciones como en el modelo. En los $7^{\circ} \mathrm{N}$ las isohalinas tienden a levantarse según se muestra en las observaciones, mientras que en el modelo las isolíneas de salinidad se situán por debajo de 10 s $50 \mathrm{~m}$ y levantamiento se corre hacia los $8^{\circ} \mathrm{N}$. El contorno de 34.8ups observado, se localiza alrededor de los $250 \mathrm{~m}$ de profundidad, mientras que en el modelo oscila entre los $200 \mathrm{~m}$ y $350 \mathrm{~m}$, notorio en todo el rango de la latitud.

La variación longitudinal de la temperatura en la capa superficial es similar a los resultados del modelo con temperaturas que rondan $\operatorname{los} 29^{\circ} \mathrm{C}$ y $28^{\circ} \mathrm{C}$. La capa de mezcla es similar a lo obtenido por Brenes et al. (2016). Luego de los $50 \mathrm{~m}$, en las observaciones (Fig. $8 \mathrm{C}$ ) y en la información del modelo (Fig. 8D), se presenta una distribución similar de temperaturas. Entre los $86^{\circ} \mathrm{W}$ y $85^{\circ} \mathrm{W}$ y $\operatorname{los} 200 \mathrm{~m}$ de profundidad 
de las obsevaciones, se distingue un núcleo de salinidad, de 34.0ups, mientras que en los resultados del reanálisis, es un núcleo más amplio en la vertical como en longitud, de 34.9ups. Alrededor de $\operatorname{los} 87.5^{\circ} \mathrm{W}$, el valor de temperatura en la superficie $\left(29^{\circ} \mathrm{C}\right)$ de las observaciones y de los datos del modelo $\left(28^{\circ} \mathrm{C}\right)$, es similar al valor de temperatura promedio reportado por
Lizano (2008) para el trimestre enero-marzo, de $28.05^{\circ} \mathrm{C}$. Para el mismo punto, el valor de salinidad en superificie de las observaciones y del modelo, es alrededor de 32.0ups a 33.0ups, comparable con el valor promedio de eneromarzo obtenido por Lizano (2008), de 32.4ups.

La Fig. 9 presenta el promedio mensual de la distribución de temperatura y salinidad
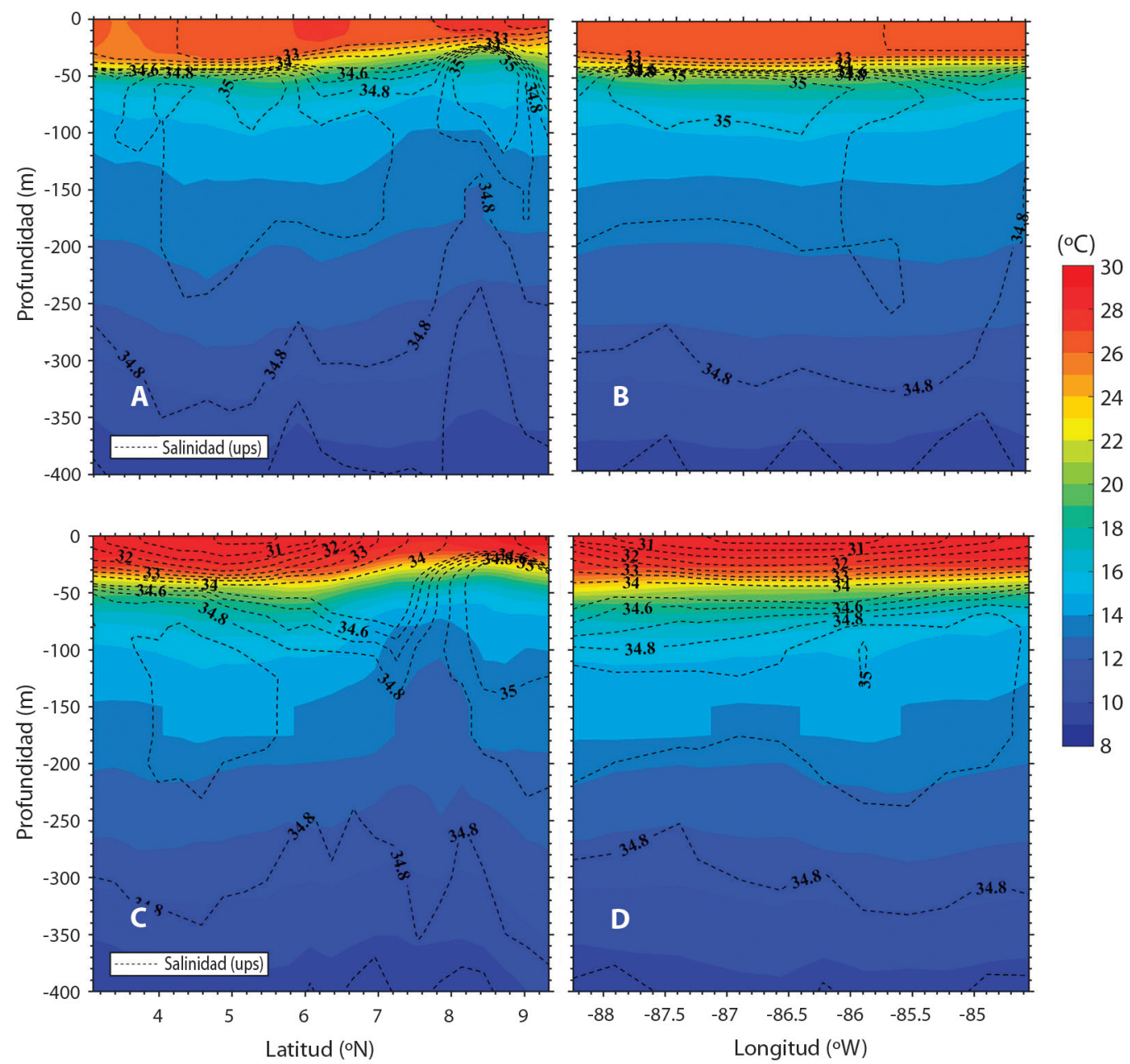

Fig. 9. Cortes verticales de temperatura y salinidad. Promedios mensuales obtenidos de la información del modelo HYCOMNCODA reanálisis. A) Corte latitudinal para octubre 2010. B) Corte longitudinal para octubre 2010. C) Corte latitudinal para marzo 2011. D) Corte longitudinal para marzo 2011. Los tonos de color representan la temperatura del océano. Los contornos con líneas segmentadas representan la salinidad. El intervalo de los contornos de salinidad es 0.5ups en el rango de 30 a 34.5 ups y de 0.1 ups en el rango de 34.5 a 35.0 ups.

Fig. 9. Monthly mean vertical sections for temperature and salinity from HYCOM-NCODA reanalysis data. A) Latitudinal cross section for October 2011. B) Longitudinal cross section for October 2010. C) Latitudinal cross section for March 2011. D) Longitudinal cross section for March 2011. The colors are for sea temperature. The dashed lines contour are for salinity. The contour interval of salinity is $0.5 \mathrm{psu}$ from 30 to $30.4 \mathrm{psu}$ and $0.1 \mathrm{psu}$ from 34.5 to $35.0 \mathrm{psu}$. 
obtenido de la información del reanálisis para octubre 2010. La distribución de temperatura promedio del modelo tiende a ser similar a la distribución de temperatura de las observaciones en los perfiles de latitud como en el perfil de longitud (Fig. 7A,C y Fig. 9A, B). Los gradientes de salinidad promedio en el modelo tienden a estar muy cerca, por debajo de la capa de mezcla hasta los 50m de profundidad, muy similar a las observaciones (Fig. 7A,C y Fig. 9A,B). Para los datos de reanálisis de marzo 2011, el promedio mensual de temperatura en la información del modelo son parecidos a la distribución de temperatura de las observaciones a lo largo del perfil latitudinal (Fig. 8A y Fig. 9C) y a lo largo de un corte longitudinal (Fig. 8C y Fig. 9D). La haloclina promedio del modelo es muy similar a las observaciones. En general la distribución de temperatura y salinidad de las observaciones en los dos meses distintos del año, tienden a tener un comportamiento promedio similar a los datos del reanálisis para los dos periodos analizados, marzo y octubre.

Para la campaña hidrográfica de julio de 2012, se hicieron tres transectos latitudinales centrados en la Isla del Coco. En la Fig. 10 se muestra el perfil vertical de temperatura y salinidad para cada uno de los transectos mostrados en la Fig. 1A (círculos negros). En la misma Fig. 10 en el lado derecho se presenta la distribución vertical de temperatura y salinidad del reanálisis. En los tres cortes latitudinales (Figs. 10A, C, E) se observa en la capa superficial la presencia de dos masas de agua distinguibles, una masa hacia el sur de la isla con temperatura de $27^{\circ} \mathrm{C}$ y otra masa hacia el norte de la isla con temperatura de $28^{\circ} \mathrm{C}$. La termoclina en las observaciones está localizada en promedio a $50 \mathrm{~m}$ de profundidad igual que en los datos del modelo. La capa superficial en los datos del modelo (Figs. 10B y $10 \mathrm{~F}$ ) tiende a tener la presencia de estas dos masas de agua similares a las observaciones. El promedio mensual de temperatura en los datos del modelo, Fig. 11B, tiende a parecerse a las observaciones de la Fig. 10C. La distribución de salinidad en el modelo (Figs. 10B, D, E) presenta mayor variabilidad en la columna de agua que en las observaciones. Las isohalinas en las observaciones están casi alineadas con la profundidad.

\section{DISCUSIÓN}

Los perfiles obtenidos durante los muestreos hidrográficos en el PTE y de los tomados alrededor de la Isla del Coco, confirman lo propuesto por Brenes (1984), Brenes et al. (1995), Badan (1997) y Brenes et al. (2016), ya que en general se observó la presencia de AST en los primeros $50 \mathrm{~m}$ de profundidad, con temperaturas superiores a $25^{\circ} \mathrm{C}$ y salinidades menores 33 psu y ASS bajo los $60 \mathrm{~m}$ con temperaturas menores a $25^{\circ} \mathrm{C}$ y salinidades alrededor de las 35 ups. La zona de transición entre ellas se presentó entre los 50-60m. En ninguno de los perfiles analizados se detectó la presencia de AIA.

En concordancia con Brenes (1985), los resultados de la temperatura del mar y la salinidad de los transectos alrededor de la Isla del Coco, reflejan las condiciones climáticas y atmosféricas observadas en la Isla del Coco (Alfaro, 2008; Alfaro \& Hidalgo, 2016; Durán \& Alfaro, 2016), en donde se observa que las variaciones estacionales en la Isla del Coco están fuertemente influenciadas por la migración meridional de la Zona de Convergencia Inter-Tropical, ya que la isla se encuentra bajo su influencia prácticamente desde la primavera hasta el otoño boreal (Quirós \& Hidalgo, 2016). En la Isla del Coco, se observa que el bimestre del año más cálido es el de marzoabril y las menores temperaturas se registran durante el segundo semestre (julio-diciembre), con temperaturas superficiales del mar entre los 27.7 y los $28.1^{\circ} \mathrm{C}$ en su promedio anual. Los ciclos anuales de registros de precipitación muestran que entre el 72 y el $75 \%$ de la precipitación acumulada anualmente se registra entre abril y octubre, con valores que rondan los 5000-7 000mm. Adicionalmente, la dirección predominante del viento es del suroeste y el periodo de mayor energía cinética es el bimestre octubre-noviembre (Alfaro, 2008). 

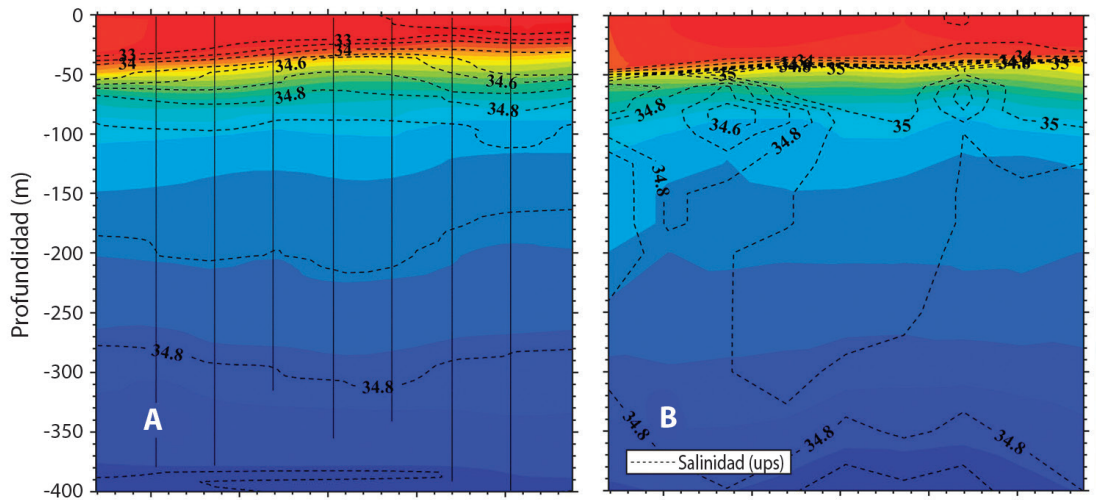

$\left({ }^{\circ} \mathrm{C}\right)$
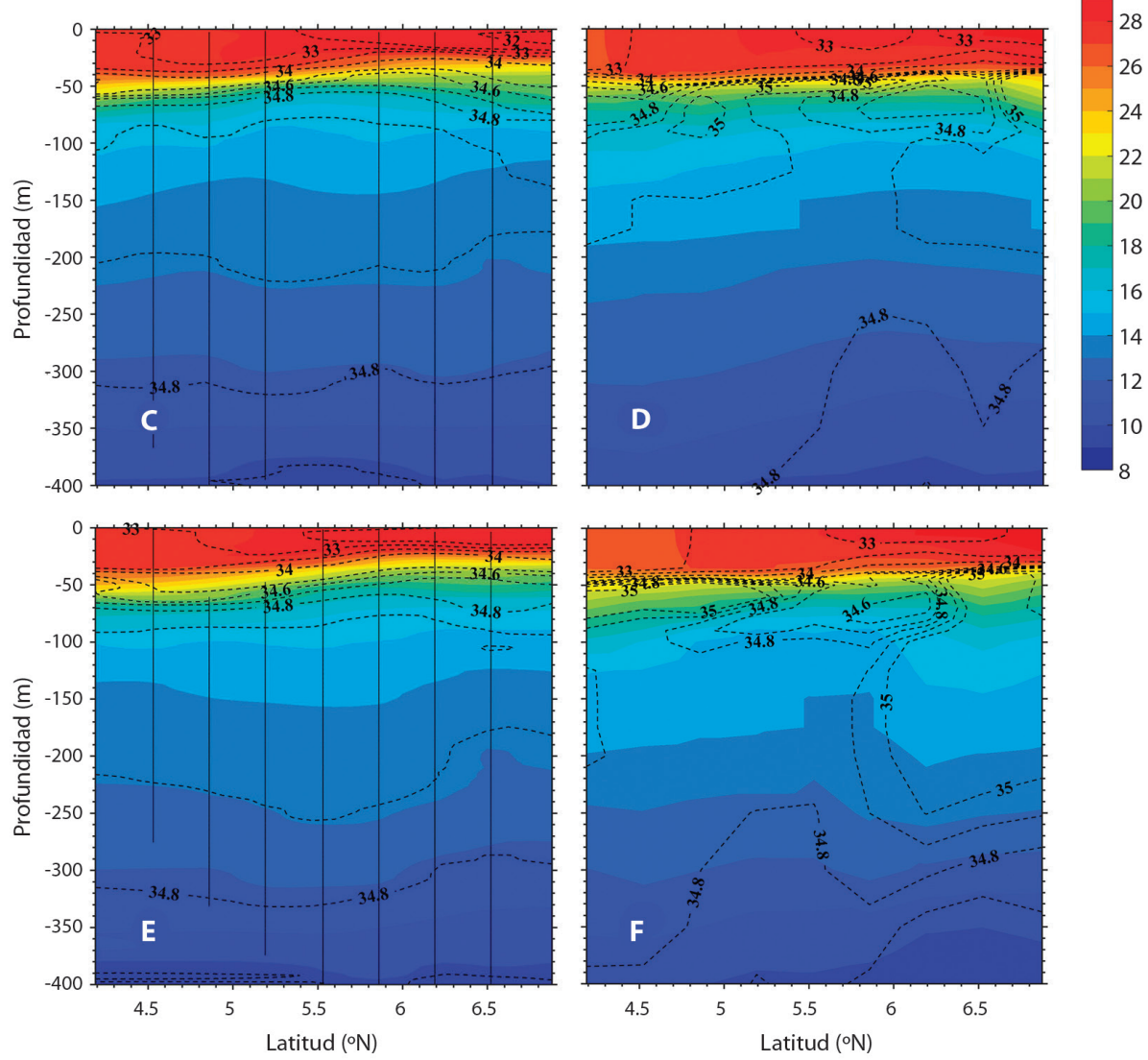

Fig. 10. Corte vertical en latitud de temperatura y salinidad para la campaña de julio de 2012. En A-C-E) observaciones usando un CTD. En B-D-F) datos del reanálisis HYCOM-NCODA. Las observaciones corresponden al transecto con círculos verdes oscuros en la Fig. 1A. Las coordenadas en longitud son: A-B) $87.72^{\circ} \mathrm{W}$, C-D) $87.05^{\circ} \mathrm{W}$ y E-F) $86.38^{\circ} \mathrm{W}$. Los tonos de color representan la temperatura del océano. Los contornos con líneas segmentadas representan la salinidad. El intervalo de los contornos de salinidad es 0.5 ups en el rango de 30 a 34.5 ups y de 0.1 ups en el rango de 34.5 a 35.0 ups. Las líneas verticales con puntos son las estaciones de muestreo.

Fig 10. Latitudinal vertical cross section for temperature and salinity, campaign of July 2012. In A-C-E) CTD casts. In B-DE) data from HYCOM-NCODA reanalysis. The observations correspond to the transect with dark green circles in Fig. 1A. The coordinates in longitude are: A-B) $87.72^{\circ} \mathrm{W}$, C-D) $87.05^{\circ} \mathrm{W}$ y E-F) $86.38^{\circ} \mathrm{W}$. The colors are for sea temperature. The dashed line contours are for salinity. The contour interval of salinity is $0.5 \mathrm{psu}$ from 30 to $30.4 \mathrm{psu}$ and $0.1 \mathrm{psu}$ from 34.5 to 35.0psu. The vertical lines with points are the cast stations. 

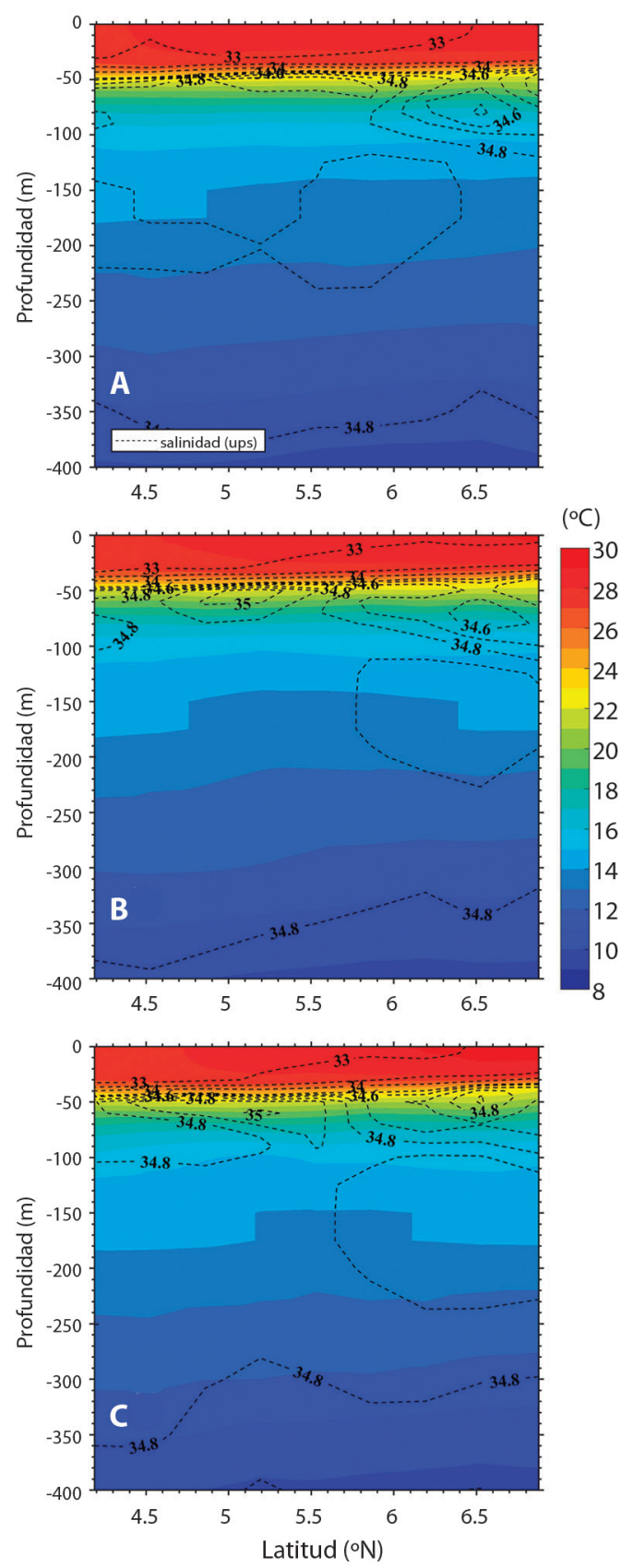

Los perfiles de las figuras 2-4, muestran valores de salinidad baja y temperatura del mar alta, propias del AST (e.g. Brenes, 1984; Brenes et al., 1995; Badan, 1997 y Brenes et al., 2016). Estos resultados concuerdan con los de Alfaro \& Hidalgo (2016). Ellos describieron que en general los datos in-situ tomados durante las expediciones realizadas a la Isla del Coco mostraron anomalías positivas de precipitación, de la temperatura superficial de aire y del mar, así como de la velocidad del viento, con algunas pequeñas excepciones descritas en Alfaro \& Hidalgo (2016). El resultado anterior muestra que, las variaciones de salinidad y temperatura del mar observados en los perfiles alrededor de la Isla del Coco, se deben principalmente a la variación estacional de la precipitación, temperatura y velocidad del viento descritas en el párrafo anterior por Alfaro (2008), Alfaro \& Hidalgo (2016) y Durán \& Alfaro (2016). Otros factores para considerar son su ubicación geográfica con respecto al viento predominante del SW sobre la isla, es decir, perfiles a barlovento y sotavento, lo cual puede ayudar o no a la mezcla vertical y el aporte de la descarga de los ríos y quebradas existentes en la isla (Díaz-Bolaños et al., 2012). Adicionalmente, Alfaro \& Hidalgo (2016) reportaron que se observaron condiciones neutras de El NiñoOscilación del Sur durante todas las campañas. Brenes, Márquez, Quirós \& Benavides (2012) identificaron también el AST y el ASS en otra región costera, cerca de Ostional, Costa Rica y sus alrededores.

Los resultados presentados en este estudio se contextualizan de acuerdo a lo descrito por Lizano (2016) quién también mostró que en un transecto norte-sur $\left(12.5-7.5^{\circ} \mathrm{N}\right.$ a lo largo del meridiano $85^{\circ} \mathrm{W}$ ), las estaciones más al norte, tienen la mayor variabilidad climatológica de

Fig. 11. Perfil vertical de temperatura y salinidad promedio para el mes de julio 2012 obtenido de la información del reanálisis HYCOM-NCODA. Corte en latitud para las coordenadas: A) $87.72^{\circ} \mathrm{W}$, B) $87.05^{\circ} \mathrm{W}$ y C) $86.38^{\circ} \mathrm{W}$. Los tonos de color representan la temperatura del océano. Los contornos con líneas segmentadas representan la salinidad. El intervalo de los contornos de salinidad es 0.5 ups en el rango de 30 a 34.5 ups y de 0.1 ups en el rango de 34.5 a 35.0 ups.

Fig. 11. Mean vertical cross section for temperature and salinity in July 2012 using HYCOM-NCODA reanalysis data. Latitudinal cross sections for the longitude: A) $87.72^{\circ} \mathrm{W}$, B) $87.05^{\circ} \mathrm{W}$ and C) $86.38^{\circ} \mathrm{W}$. The colors are for sea temperature. The dashed line contours are for salinity. The contour interval of salinity is $0.5 \mathrm{psu}$ from 30 to $30.4 \mathrm{psu}$ and $0.1 \mathrm{psu}$ from 34.5 to $35.0 \mathrm{psu}$. 
los perfiles de temperatura y salinidad, lo cual es coherente con la mayor variabilidad de los enfriamientos superficiales que se dan al norte de $\operatorname{los} 9.5^{\circ} \mathrm{N}$. Sobre estas estaciones se puede encontrar una capa de mezcla de temperatura y salinidad, en los primeros meses del año (enero a abril) (Lizano, 2016), lo cual está relacionado con la intensidad de los vientos que se generan durante estos meses (Amador et al., 2006; Alfaro \& Cortés, 2012; Alfaro et al., 2012). En los meses de abril, julio y octubre, en estas estaciones también se pueden encontrar inversiones en el gradiente de salinidad con profundidad, lo cual puede estar relacionado con la evaporación de las aguas superficiales (Knauss, 1978). La distribución de la salinidad en la capa superficial de todas las estaciones estudiadas por Lizano (2016), es menor que la salinidad sobre la capa profunda, lo cual está relacionado a las altas precipitaciones que se dan sobre esta parte del PTE (Amador et al., 2006; 2016a; b). Además, se sabe que esta es una de las zonas de menor salinidad de todos los océanos tropicales del mundo (Lizano, 2008; 2016).

Los perfiles hidrográficos realizados en el PTE (Figs. 7A y 7C) en el mes de octubre 2010 y marzo 2011 están dentro del rango climatológico de temperatura y salinidad en los primeros $10 \mathrm{~m}$ de profundidad obtenidos por Lizano (2016), para el mes de octubre con temperaturas entre 26 y $27^{\circ} \mathrm{C}$ y salinidades alrededor de los 32 y 33 ups, mientras para el mes de marzo los muestreos hidrográficos tienden a parecerse a la climatología de abril (Lizano, 2016), con aguas más cálidas respecto al perfil de octubre, salinidades de 33ups alrededor del transecto longitudinal sobre la Isla del Coco y de 33.5ups o más hacia el continente. A nivel de superficie los resultados de los perfiles hidrográficos del mes de marzo 2011, de temperatura y salinidad, son similares a los valores promedio de eneromarzo de temperatura superficial obtenido por Lizano (2008). Estos resultados obtenidos a través de los perfiles oceanográficos en los alrededores de la Isla del Coco y el PTE confirman que tanto el conjunto de datos utilizado en Lizano (2008) y Lizano (2016) pueden ser usados para caracterizar la región.
Los resultados de los perfiles oceánicos de temperatura en el PTE son similares a los resultados mostrados en el estudio de Brenes et al. (2016), en el transecto latitudinal de octubre se presenta una capa de mezcla que se profundiza hasta los $50 \mathrm{~m}$ y con un levantamiento de la termoclina hacia el norte del perfil, mientras para el transecto longitudinal de octubre la capa isotérmica es más homogénea en todo el corte, posiblemente debido a la presencia establecida de la Contracorriente Ecuatorial (Kessler, 2006) sobre la isla. Para los transectos hidrográficos realizados en marzo, estos son comparables con los obtenidos por Brenes et al. (2016), con un levantimiento más evidente de la termoclina hacia el norte del transecto latitudinal relacionado con los fuertes vientos que se da en la región de los Papagayos (Amador et al., 2006; Alfaro \& Cortés, 2012; Alfaro et al., 2012). Es importante destacar, de los resultados del transecto longitudinal de temperatura y salinidad, que no hay evidencia de un giro ciclónico como lo reporta Brenes et al. (2016) hacia el oeste de la Isla del Coco.

En cuanto a los muestreos hidrográficos realizados en julio 2012, los perfiles de temperatura y salinidad en los primeros metros de profundidad son similares a los resultados obtenidos por Lizano (2016), con temperaturas entre los 27 y $28^{\circ} \mathrm{C}$ y salinidades entre los 33 y 34 ups. Estos valores altos están relacionados con el veranillo que se presenta en este mes en la región centroamericana y que se extiende hasta la Isla del Coco (Amador et al., 2006; 2016a; b), donde se presentan anomalias positivas de temperatura superficial del mar lo que genera un mayor flujo de calor latente (Alfaro, 2008; Alfaro \& Hidalgo, 2016).

Finalmente, el estudio mostró que los datos del reanálisis del modelo de circulación general HYCOM-NCODA son comparables con los resultados de los perfiles del oceanográficos del CTD en el PTE. La distribución de la temperatura en profundidad de los muestreos tiende a ser similar a los datos del modelo oceánico y las principales diferencias se dan en la capa profunda para la salinidad, por lo que el modelo HYCOM es capaz de reproducir las 
características observadas en el PTE de temperatura y salinidad y puede ser usado para estudiar la dinámica tropical oceánica, en regiones más extensas y en otras épocas del año.

Declaración de ética: los autores declaran que todos están de acuerdo con esta publicación y que han hecho aportes que justifican su autoría; que no hay conflicto de interés de ningún tipo; y que han cumplido con todos los requisitos y procedimientos éticos y legales pertinentes. Todas las fuentes de financiamiento se detallan plena y claramente en la sección de agradecimientos. El respectivo documento legal firmado se encuentra en los archivos de la revista.

\section{AGRADECIMIENTOS}

Se agradece a los siguientes proyectos inscritos en la Vicerrectoría de Investigación, Universidad de Costa Rica: 808-B5-298, B0-654 (apoyado por FEES-CONARE), A9-902, A7-520 (apoyado por el FFEM), 805-B8-766 (apoyado por el Fondo de apoyo a Redes Temáticas de la Universidad de Costa Rica), B9-454 (apoyado por el Fondo de Grupos de la Universidad de Costa Rica), B7-286 (apoyado por el Programa de Estudios Avanzados de la Universidad de Costa Rica, UCREA), B7-507 (apoyado por CONICIT y MICITT) y A1-715. Las gracias a Mauricio Lao Escalante por su apoyo en la edición de las imágenes. A los revisores anónimos por sus valiosos comentarios.

\section{RESUMEN}

Introducción: Para Costa Rica, los estudios realizados en el Pacífico Tropical Este o PTE son importantes ya que se relacionan con el Corredor Marino del Pacífico Este Tropical, el cual es un esfuerzo de conservación multinacional. El presente estudio describe los parámetros físicos de temperatura del mar y salinidad del océano de las masas de agua que rodean a la Isla del Coco y su entorno, el PTE, a través del análisis de perfiles de CTD obtenidos durante campañas hidrográficas realizadas entre el año 2008 y el 2012; y comparar los resultados de los datos in-situ con los del reanálisis del modelo HYCOM-NCODA. Métodos: Se compilaron los resultados de 8 diferentes campañas científicas a la Isla del Coco de abril de 2008, marzo de 2009, abril de 2010, julio de 2011 y marzo de 2012. También se analizan las campañas de octubre de 2010 y marzo de 2011 en donde se realizó un transecto latitudinal y un transecto longitudinal en las aguas del Pacífico Tropical Este de Costa Rica. En julio de 2012 se realizaron 3 transectos latitudinales en las aguas en torno a la Isla del Coco. Las mediciones se hicieron por medio de un CTD, instrumento utilizado para determinar la conductividad, temperatura y presión de la columna de agua, entre otras variables. Resultados: Se observó la presencia de Agua Superficial Tropical en los primeros $50 \mathrm{~m}$ de profundidad, con temperaturas superiores a $25^{\circ} \mathrm{C}$ y salinidades menores 33 psu y Agua Sub-superficial Sub-tropical bajo los $60 \mathrm{~m}$ con temperaturas menores a $25^{\circ} \mathrm{C}$ y salinidades alrededor de las 35 ups. La zona de transición entre ellas se presentó entre los 50-60m. Los resultados de la temperatura del mar y la salinidad de los transectos analizados reflejan las condiciones climáticas y atmosféricas observadas, en donde se nota que las variaciones estacionales en el PTE están fuertemente influenciadas por la migración meridional de la Zona de Convergencia Inter-Tropical. Conclusiones: El estudio mostró que los datos del reanálisis del modelo de circulación general HYCOM-NCODA son comparables con los resultados de los perfiles del CTD en el PTE. La distribución de la temperatura de los muestreos tiende a ser similar a los datos del modelo oceánico y las principales diferencias se dan en la capa profunda para la salinidad, por lo que el modelo HYCOM es capaz de reproducir las características observadas en el PTE de temperatura y salinidad y puede ser usado para estudiar la dinámica tropical oceánica, en regiones más extensas y en otras épocas del año.

Palabras clave: temperatura del mar, salinidad, distribución vertical, perfiles de CTD, Isla del Coco, Pacífico Tropical Oriental.

\section{REFERENCIAS}

Alfaro, E. (2008). Ciclo diario y anual de variables troposféricas y oceánicas en la Isla del Coco, Costa Rica. Revista de Biología Tropical, 56(Supplement 2), 19-29.

Alfaro, E., \& Cortés, J. (2012). Atmospheric forcing of cool subsurface water events in Bahía Culebra, Gulf of Papagayo, Costa Rica. Revista de Biología Tropical, 60(Supplement 2), 173-186.

Alfaro, E. J., Cortés, J., Alvarado, J. J., Jiménez, C., León, A., Sánches-Noguera, C., Nivia-Ruiz, J., \& Ruiz, E. (2012). Clima y temperatura superficial del mar en Bahía Culebra, Golfo de Papagayo, Costa Rica. Revista de Biología Tropical, 60(Supplement 2), 159-171.

Alfaro, E., \& Hidalgo, H. (2016). Climate of an oceanic island in the Eastern Pacific: Isla del Coco, Costa Rica, Central America. Revista de Biología Tropical, 64(Supplement 1), S59-74. 
Amador, J. A., Alfaro, E. J., Lizano, O. G., \& Magaña, V. (2006). Atmospheric forcing in the Eastern Tropical Pacific. Progress in Oceanography, 69, 101-142.

Amador, J. A., Rivera, E. R., Durán-Quesada, A. M., Mora, G., Sáenz, F., Calderón, B., \& Mora, N. (2016a). The easternmost tropical Pacific. Part I: A climate review. Revista de Biología Tropical, 64(Supplement 1), S1-22.

Amador, J. A., Durán-Quesada, A. M., Rivera, E. R., Mora, G., Sáenz, F., Calderón, B., \& Mora, N. (2016b). The easternmost tropical Pacific. Part II: Seasonal and intraseasonal modes of atmospheric variability. Revista de Biología Tropical, 64(Supplement 1), S23-57.

Badan, A. (1997). La corriente costera de Costa Rica en el pacífico mexicano. En M. Lavín (Ed.), Contribuciones a la Oceanografía Física en México (pp. 99-112). Monografía No. 3, Unión Geofísica Mexicana.

Ballestero D., \& Coen, J. E. (2004). Generation and propagation of anticyclonic rings in the Gulf of Papagayo. International Journal of Remote Sensing, 25(11), 2217-2224

Barton, E. D., Lavin, M. F., \& Trasviña, A. (2009). Coastal circulation and hydrography in the Gulf of Tehuantepec, Mexico, during winter. Continental Shelf Research, 29(2), 485-500.

Brenes, C. (1984). Las masas de agua del Oceano Pacífico Tropical frente a Costa Rica. Uniciencia, 1(1), 31-36.

Brenes, C. (1985). Condiciones de movimiento geostrófico para la corriente costera de Costa Rica. Uniciencia, 2(1), 21-27.

Brenes, C., Ballestero, D., Benavides, R., Salazar, J. P, \& Murillo, G. (2016). Variation in the geostrophic circulation pattern and thermohaline structure in the Southeast Central American Pacífic. Revista de Biología Tropical, 64(Supplement 1), 121-S134.

Brenes, C., \& Coen, J. E. (1985). Correlación T-S de las masas de agua en la región del Domo Térmico de Costa Rica. Uniciencia, 2(1), 42-50.

Brenes, C., Kwiecinski, B., D’Croz, L., \& Chávez, J. (1995). Características oceanográficas de la plataforma pacífica de América Central y aguas oceánicas adyacentes. Programa Regional de Apoyo al Desarrollo de la Pesca en el Istmo Centroamericano (PRADEPESCA).

Brenes, C., Márquez, A., Quirós, W., \& Benavides, R., (2012). Rasgos hidrográficos y batimétricos del Refugio Nacional de Vida Silvestre Ostional y áreas adyacentes, Guanacaste, Costa Rica. Revista Ciencias Marinas y Costeras, 4, 145-156.

Broenkow, W. (1965). The distribution of nutrients in the Costa Rica Dome in the eastern tropical Pacific
Ocean. Limnology and Oceanography, 10(1), 40-52, doi: 10.4319/lo.1965.10.1.0040.

Chassignet, E. P., Hurlburt, H. E., Metzger, E. J., Smedstad, O. M., Cummings, J. A., Halliwell, G. R., Bleck, R., Baraille, R., Wallcraft, A. J., Lozano, C., Tolman, H. L., Srinivasan, A., Hankin, S., Corinillon, P., Weisberg, R., Barth, A., He, R., Werner, F., \& Wilkin, J. (2009). US GODAE: global ocean prediction with the HYbrid Coordinate Ocean Model (HYCOM). Oceanography, 22(2), 64-75.

Cortés, J., Morales, A., Alfaro, E., Lizano, O., \& Acuña, J. (2009). Conocimiento y gestión de medios marinos y coralinos del Área de Conservación Marina Isla del Coco, Costa Rica en el marco del Proyecto Protección de la Biodiversidad del Área de Conservación Marina Isla del Coco - SINAC/FFMAM, Informe Final. Recuperado de http://www.kerwa.ucr.ac.cr/ handle/10669/436

Cortés, J., Brenes, C., Morales, A., Lizano, O., Alfaro, E., Acuña, J., Ballestero, D., \& Moreno, M. (2012). Interacciones océano-atmósfera y la biodiversidad marina del Parque Nacional Isla del Coco, Costa Rica, Informe Final. Recuperado de http://www.kerwa.ucr. ac.cr/handle/10669/11379

Cummings, J. A. (2005). Operational multivariate ocean data assimilation. Quarterly Journal of the Royal Meteorological Society, 131, 3583-3604.

Díaz-Bolaños, R. D., Alfaro, E. J., \& Ugalde-Quesada, A. (2012). Diccionario histórico-toponímico de la Isla del Coco, Costa Rica. Revista de Biología Tropical, 60(Supplement 3), 1-14.

Durán, A. M, \& Alfaro, E. (2016). A multi-scale analysis of moisture supply associated with precipitation on Isla del Coco, Pacific Costa Rica. Revista de Biología Tropical, 64(Supplement 1), S87-103.

Fiedler, P. C. (2002). The annual cycle and biological effects of the Costa Rica Dome. Deep-Sea Research I, 49, 321-338.

Fiedler, P. C., \& Talley, L. D. (2006). Hydrography of the Eastern Tropical Pacific: A review. Progress in Oceanography, 69, 143-180.

Flores-Vidal, X., Durazo, R., Chavanne, C., \& Flament, P. (2011). Coastal circulation in the absence of wind in the Gulf of Tehuantepec, Mexico: High-frequency radar observations. Ciencias marinas, 37(4A), 493-512.

Hidalgo, H. G., Durán-Quesada, A. M., Amador J. A., \& Alfaro, E. J. (2015). The Caribbean Low-Level Jet, the Inter-Tropical Convergence Zona and the precipitation patterns in the Intra-Americas Sea: A proposed dynamical mechanism. Geografiska Annaler, Series A: Physical Geography, 97, 41-59. doi: 10.1111/ geoa.12085. 
Kessler, W. S. (2006). The circulation of the eastern tropical Pacific: A review. Progress in Oceanography, 69, 181-217.

Knauss, J. A. (1978). Introduction to Physical Oceanography. Nueva York: Prentice-Hall.

Lizano, O.G. (2001). Batimetría de la plataforma insular alrededor de la Isla del Coco, Costa Rica, Centro América. Revista de Biología Tropical, 49(Supplement 2), 163-170.

Lizano, O. G. (2008). Dinámica de las aguas alrededor de la Isla del Coco, Costa Rica. Revista de Biología Tropical, 56(Supplement 2), 31-48.

Lizano, O. G. (2013). Erosión en las playas de Costa Rica, incluyendo la Isla del Coco. Revista InterSedes, 27(14), 6-27.

Lizano, O. G. (2016). Distribución espacio-temporal de la temperatura, salinidad y oxígeno disuelto alrededor del Domo Térmico de Costa Rica. Revista de Biología Tropical, 64(Supplement 1), S135-S152.

Palacios, D. M., \& Bograd, S. J. (2005). A census of Tehuantepec and Papagayo eddies in the northeastern tropical Pacific. Geophysical Research Letters, $32(23)$.

Peñaherrera-Palma C., Arauz, R., Bessudo, S., BravoOrmaza, E., Chassot, O., Chinacalle-Martínez, N., Espinoza, E., Forsberg, K., García-Rada, E., Guzmán, H., Hoyos, M., Hucke, R., Ketchum, J., Klimley, A.P., López-Macías, J., Papastamatiou, Y., Rubin, R., Shillinger, G., Soler, G., Steiner, T., Vallejo, F., Zanella, I., Zárate, P., Zevallos-Rosado, J. \& Hearn, A. (2018). Justificación biológica para la creación de la MigraVia Coco-Galápagos. MigraMar y Pontificia Universidad Católica del Ecuador Sede Manabí. Portoviejo, Manabí, Ecuador. Recuperado de http:// migramar.org/hi/es/publicaciones-2/
Quirós, E. (2015). Variabilidad climática de la Zona de Convergencia Intertropical y su influencia en la hidrometeorología centroamericana. (Tesis de Maestría). Universidad de Costa Rica, San José, Costa Rica.

Quirós, E., \& Hidalgo, H. G. (2016). Interacción de la Zona de Convergencia Intertropical del Pacífico Este con Sistemas Tropicales. Tópicos Meteorológicos y Oceanográficos, 15(2), 33-47.

Reyes-Hernández, C., Ahumada-Sempoal, M. Á., \& Durazo, R. (2016). The Costa Rica coastal current, eddies and wind forcing in the Gulf of Tehuantepec, southern Mexican Pacific. Continental Shelf Research, 114, 1-15.

Trasviña, A., Barton, E. D., Brown, J., Velez, H. S., Kosro, P. M., \& Smith, R. L. (1995). Offshore wind forcing in the Gulf of Tehuantepec, Mexico: The asymmetric circulation. Journal of Geophysical Research: Oceans, 100(C10), 20649-20663.

Trasviña, A., \& Barton, E. D. (2008). Summer circulation in the Mexican tropical Pacific. Deep Sea Research Part I: Oceanographic Research Papers, 55(5), 587-607.

Willett, C. S., Leben, R., \& Lavín, M. F. (2006). Eddies and mesoscale processes in the eastern tropical Pacific: A review. Progress in Oceanography, 69, 218-238.

Wyrtki, K. (1964). Upwelling in the Costa Rica Dome. Fishery Bulletin, 63, 355-372.

Wyrtki, K. (1966). Oceanography of the eastern equatorial Pacific Ocean. Oceanography and Marine Biology Annual Review, 4, 33-68.

Wyrtki, K. (1967). Circulation and water masses in the eastern equatorial Pacific Ocean. International Journal of Oceanology and Limnology, 1, 117-147. 